\title{
GBAS Ground Monitoring Requirements from an Airworthiness Perspective
}

\author{
Michael Felux*, Jiyun Lee ${ }^{+}$, Florian Holzapfel ${ }^{\#}$
}

* German Aerospace Center (DLR), Oberpfaffenhofen, 82234 Wessling, Germany, e-mail: michael.felux@dlr.de, phone: +49-8153-28-2993, fax: +49-8153-28-2328 (corresponding author),

${ }^{+}$Korea Advanced Institute of Science and Technology (KAIST), 291 Daehak-ro.

Daejeon 305-701, Republic of Korea

\# Technische Universität München, Lehrstuhl für Flugsystemdynamik, Boltzmannstrasse 15, 85748 Garching bei München, Germany

\section{Abstract}

The Ground Based Augmentation System (GBAS) provides corrections for satellite navigation signals together with integrity parameters to aircraft and enables precision approach guidance. It will eventually replace the currently used Instrument Landing System (ILS). GBAS Approach Service Type (GAST) C stations supporting CAT-I operations have been fully developed and certified and first stations are operational. For the service type D, which is intended to support CAT-III operations including automatic approaches and landings, requirements have been drafted and are currently undergoing validation. One remaining issue is the requirement for monitoring of ionospheric anomalies in the ground subsystem. Large gradients in the concentration of free electrons in the ionosphere can lead to significant positioning errors when navigation is based on differential methods. We give a review of the derivation of currently proposed performance requirements for such a monitor. Next, we show that the required level of safety from an airworthiness perspective can be achieved even with relaxed monitoring requirements compared to the currently drafted standards. These relaxations result from satellite geometry assessments on the ground and actual approach characteristics towards a runway. We show that with this method it is sufficient to monitor for gradients in the range of about $450-550 \mathrm{~mm} / \mathrm{km}$ while current standards require detection already from $300 \mathrm{~mm} / \mathrm{km}$. A remote monitoring receiver near the touchdown point can monitor the post-correction differential range error and use it as test statistic for GBAS performance monitoring and protection against ionospheric disturbances.

\section{Keywords}

GBAS, Ionosphere monitoring, airworthiness assessment 


\section{Introduction and background}

In March 2012 the first GBAS Approach Service Type C (GAST C) ground station achieved full certification. This service type supports operations equivalent to a CAT-I ILS with a minimum decision height of $200 \mathrm{ft}$ and a runway visual range of at least $550 \mathrm{~m}$. It is located in Bremen (ICAO identifier EDDW) in northern Germany and since then is regularly used by Air Berlin which has equipped a large portion of their B737-NG fleet with the GBAS Landing System (GLS). GBAS stations in Newark and Houston have become operational as well and are used by United Airlines with their B787 fleet and part of their B737-NG fleet. Other airports like Zurich and Frankfurt are currently installing systems. A number of trial GBAS stations with different levels of progress towards certification have been set up in several countries including Spain, France, Australia, Germany and Russia. After having reached this important milestone of GAST C certification for ground stations, current research and development effort is focusing on meeting all necessary requirements to support operations also under CAT-II/III weather conditions. The set of airborne and ground requirements for these operations based on single frequency GPS navigation is summarized as GAST D. With the main concern being decorrelation of ionospheric effects between the ground station and a user, most of the additional monitoring built into the GAST D architecture is dedicated to detection and mitigation of this threat (Murphy 2006). A potentially hazardous situation is shown in Figure 1 which illustrates a worst case ionospheric front situation.

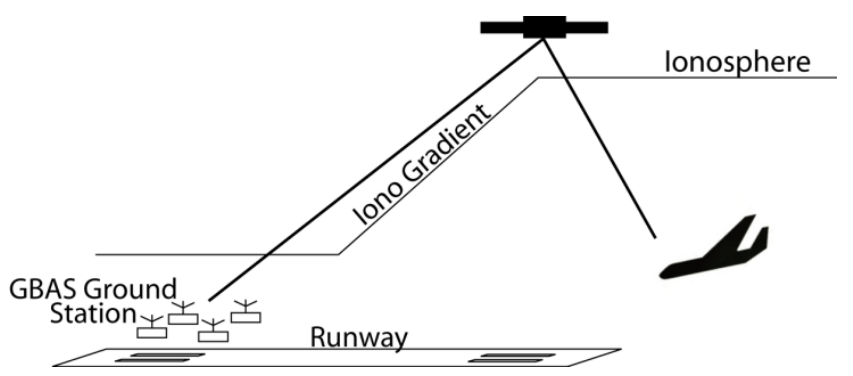

Fig. 1 Illustration of a potentially hazardous situation caused by an ionospheric front

In the Contiguous United States (CONUS) the currently valid threat model assumes a largest value for the spatial decorrelation of $425 \mathrm{~mm} / \mathrm{km}$ in pseudoranges while the German threat model only considers $140 \mathrm{~mm} / \mathrm{km}$ based on measurements over one solar cycle (Pullen 2009; Mayer 2009). Despite the fact that those threat models have been established, long-term monitoring is still on-going to validate these values (Jung 2012).

A GBAS ground station is required to protect users from potentially dangerous ionospheric situations. Gradients up to $300 \mathrm{~mm} / \mathrm{km}$ are accounted for in the protection level risk together with a siting limit which restricts the largest distance between the GBAS reference point and a runway threshold to $5 \mathrm{~km}$. Larger gradients 
have to be identified by an ionospheric gradient monitor. There have been two proposals how to address this task. The first one uses double-differenced carrier phase measurements (Khanafseh 2012). This method, however, requires a pre-defined prior probability of occurrence of an ionospheric anomaly and antenna phase center stability in the order of millimeters. The $5 \mathrm{~km}$ siting limit is necessary with this architecture to protect users from the effect of worst case gradients. The second method is a carrier phase based, code aided technique (Fujita 2010). It is based on resolving carrier phase ambiguities, but correct fixing of the integer ambiguity with the required level of integrity is still a challenge.

In the next section we propose a different approach to this task by reviewing the origin of the currently proposed requirements and suggesting possible relaxations at certain steps where unnecessarily conservative assumptions are made. At all times the safety targets are maintained but instead of taking worst case assumptions, the actual approach geometry as well as the current satellite geometry are considered. In the following two sections we present a scheme for ground monitoring which applies these relaxations and is designed to fulfill the ionosphere monitoring task. Finally, an investigation of the monitor performance is carried out for various potential GBAS locations showing that the proposed architecture can protect users at the required level of integrity.

\section{Derivation of Ground Monitoring Requirements}

After initial attempts to derive requirements for GBAS from the performance of current ILS, the adopted strategy is to derive requirements from an airworthiness point of view (Schuster 2010). A schematic overview is given in Figure 2.

(1)

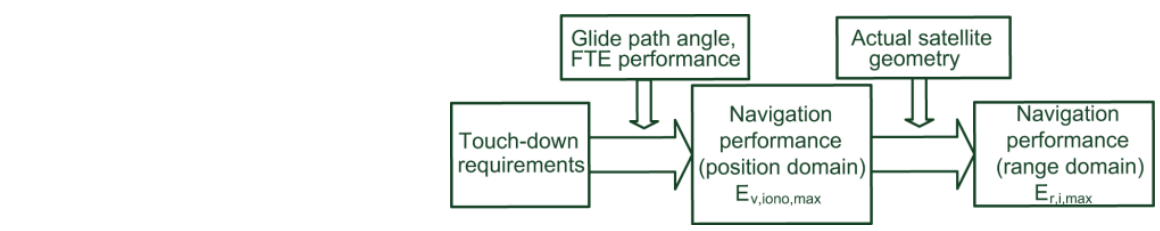

Fig. 2 Derivation of range-domain requirements from airworthiness considerations

A limit on the largest vertical positioning error is determined from the touch-down requirements. It is sufficient to consider only the vertical requirements since they are more difficult to fulfill than the lateral ones. This is done making assumptions about the performance of autopilot systems and the glide path angle of the approach. The proposed monitor would not be operating in the position domain, but rather in the range domain and thus depend on the actual current satellite geometry. We now discuss the way how the thresholds are derived and 
propose changes to the assumptions where appropriate, in order to relax the requirements without compromising safety. The derivation and assumptions follow the steps described by Burns (2009).

The drafted and frozen standards and recommended practices (SARPs) (ICAO 2010) result from the definition of a safe landing, as given in European certification specifications for all-weather operations CS-AWO 131 (EASA 2003) and similarly in AC 120-28D (FAA 1999). The most relevant part for GBAS relates to the requirement that the aircraft lands inside the so-called touchdown box with a sensible attitude and sink rate. It is an area on the runway where the main landing gear of the aircraft has to touch down. The longitudinal dimensions and tolerable probabilities of not meeting the requirement are summarized in Table 1 and depicted in Figure 3. All distances are given in direction of flight behind the runway threshold. The lateral limit is $5 \mathrm{ft}$ (1.5 m) from the edge of the runway for the outer main landing gear.

Table 1 Touchdown requirements according to EASA (2003) and FAA (1999)

\begin{tabular}{|c|c|c|c|}
\hline Case & Land short limit (ft) & Land long limit (ft) & $\begin{array}{c}\text { Allowable probability of } \\
\text { exceeding the limit }\end{array}$ \\
\hline \hline Nominal & 200 & 2700 & $10^{-6}$ \\
\hline Limit & 200 & 3000 & $10^{-5}$ \\
\hline Malfunction & 200 & 3000 & 0 \\
\hline
\end{tabular}

CS-AWO distinguishes three different cases for which the aircraft has to land in the touchdown box: the nominal case, the limit case, and the malfunction case. In the nominal case all influencing parameters vary according to their nominal distributions. In the limit case one parameter is kept at its most critical value while all other parameters vary according to their nominal distributions. In the malfunction case, one parameter exceeds its threshold value without detection or mitigation within the system. An undetected ionospheric gradient resulting in large positioning errors can be treated as a malfunction. Further discussion is thus limited to this case. In the malfunction case the aircraft has to land in the touchdown box with complete certainty for any effect occurring and not being detected with a higher probability than $10^{-9}$. 

position error $E_{v}$ on the approach would translate to an along-track touchdown error $E_{\text {art }}$ which is the relevant parameter in terms of the requirements mentioned. The relation is described based on the glide path angle (GPA) of the approach as:

$$
E_{v}=E_{\text {artk }} \cdot \tan (G P A)
$$

113 This description originates from the autopilot logic which switches vertical guidance from ILS or GLS to the radar altimeter at a defined radar altitude, typically between 30 and $100 \mathrm{ft}$ depending on the aircraft. Glide path angles of precision approaches can be in the range of $2.5^{\circ}$ to $3.5^{\circ}$. For a conservative assessment of the maximum allowable vertical error, a GPA of $2.5^{\circ}$ is assumed. This is usually unnecessarily conservative, since most approaches have a $3^{\circ}$ or even higher GPA. In any case, it is a known parameter which is transmitted in the type 4 GBAS message (RTCA 2008a) and can be used for the derivation of a monitoring requirement for each individual GBAS approach.

The largest allowable error in along-track direction is given by the land short and long limits summarized in Table 1 and the assumption that the nominal touchdown point (NTDP) is located at a distance of $1290 \mathrm{ft}(393.2 \mathrm{~m})$ behind the runway threshold. The TSE budget, however, has to be split between the Navigation System Error (NSE) and the Flight Technical Error (FTE). The assumption used in the derivation of requirements is that they are statistically independent with a standard deviation of the along-track touchdown dispersion due to the flight technical error of $\sigma_{F F E}=180 \mathrm{ft}(54.9 \mathrm{~m})$. A study about the touchdown performance was conducted by Boeing (2005) and this value is expected to apply to all aircraft types which are likely to be equipped with GLS. The NSE budget must contain the nominal GBAS noise plus an undetected vertical error $E_{v, \text { iono }}$ due to the ionosphere. The standard deviation of the fault-free vertical NSE $\sigma_{\text {NSE, vert,ff }}$ for GBAS is limited by the Vertical Alert Limit (VAL) and the associated integrity risk of $10^{-7}$ such that

$$
\sigma_{\text {NSE, vert, ff }} \leq V A L / K_{\text {ffnd }}=10 \mathrm{~m} / 5.81=1.72 \mathrm{~m}
$$

The $K_{\text {ffmd }}$ is based on three reference receivers according to Table 2-16 in DO-253C. Since the nominal NSE and FTE are usually described as Gaussian distributed random variables, complete certainty cannot be achieved unless the stochastic nature is not considered. To show compliance with the requirement, fixed, non-variable values for the FTE and NSE are used. What is done in the requirement derivation and shown to be sufficient by Clark (2006) in this context is taking NSE and FTE at their $95^{\text {th }}$ percentiles corresponding to 1.96 times their standard deviation for the fault-free case (indicated as subscript ff,95\%). With the condition that the aircraft has 
to land not less than $200 \mathrm{ft}(61.0 \mathrm{~m})$ behind the runway threshold, the condition for the land-short case can be formulated as

$$
61 \mathrm{~m} \leq \mathrm{NTDP}-\frac{N S E_{\text {vert }, f f, 95 \%}+E_{\mathrm{v}, \text { iono }}}{\tan (G P A)}-\mathrm{FTE}_{\mathrm{ff}, 95 \%}
$$

With all the above described values solving (3) for $E_{v, \text { iono }}$ yields a maximum tolerable value of $E_{v, \text { iono, } \max }=6.44 \mathrm{~m}$. However, changing the GPA to a value of $3^{\circ}$ results in $E_{v, \text { iono, } \max }=8.40 \mathrm{~m}$.

Now this limit in the position domain has to be transformed into a limit in the range domain. The relation between the two domains is given by the projection matrix $S$ which is defined as

$$
S=\left(G^{T} \cdot W \cdot G\right)^{-1} \cdot G^{T} \cdot W
$$

The geometry matrix $G$ contains the normalized line-of-sight vectors from each of the $N$ satellites to the user and a " 1 " in the fourth column for the receiver clock offset. The weighting matrix $W$, as defined in DO-253C (RTCA 2008b), is a diagonal matrix containing the inverse of the expected fault-free variance of the pseudorange measurement associated with each satellite as non-zero elements. These variances $\sigma_{i}^{2}$ are given as

$$
\sigma_{i}^{2}=\sigma_{p r, g n d, i}^{2}+\sigma_{p r, a i r, i}^{2}+\sigma_{\text {tropo }, i}^{2}+\sigma_{\text {iono }, i}^{2}
$$

where $\sigma_{p r, g n d}$ describes the standard deviation of the ground station contribution, $\sigma_{p r, a i r}$ the airborne contribution due to noise and airframe multipath, $\sigma_{\text {tropo }}$ the residual tropospheric uncertainty and $\sigma_{\text {iono }}$ the residual ionospheric uncertainty (McGraw 2000, RTCA 2008b). The residual ionospheric and tropospheric uncertainties depend on the horizontal and vertical distance between the GBAS station and the user, while the other two parameters depend on the quality classification of the ground station and airborne receiver and the smoothing time. Thus, for calculation of the individual error contributions assumptions have to be made. In this study the Ground Accuracy Designator (GAD) and Airborne Accuracy Designator (AAD) were chosen as "C" and "B", respectively, in order to represent the expected equipment classification for GAST-D operations. For the ionospheric uncertainty a distance to the GBAS reference point of $6 \mathrm{~km}$ was assumed due to the $5 \mathrm{~km}$ siting limit between the reference point and the threshold plus $1 \mathrm{~km}$ for the distance between the aircraft at $200 \mathrm{ft}$ and the threshold. The position estimation is accomplished by a linearization around an initial position estimate and then a standard iterative least-squares solution which can be described as

$$
\Delta x=S \cdot \Delta y
$$

where $\Delta x$ is the 4-dimensional position and receiver clock increment for an initial position and $\Delta y$ the Ndimensional vector containing difference between the corrected and smoothed pseudoranges and the expected 
ranges based on the position estimate. The contribution of one satellite $i$ to the position estimate vertical to the approach track is given by

$$
S_{\text {vert }, i}=S_{3, i}+S_{1, i} \cdot \tan (G P A)
$$

where $S_{\text {vert }, i}$ is a scalar parameter describing the weight which is given to the measurement of satellite $i, s$ are the elements of $S$ from (6) and the coordinates are given in an approach coordinate system moving with the aircraft along the approach track. In the airborne geometry screening process in GAST-D the $S_{\text {vert }, i}$ are assumed to be limited to a maximum of 4 (Harris 2007), i.e. the contribution of a single satellite to a vertical position error with respect to the approach track cannot be larger than 4 times the corresponding post correction range error. Hence, according to the draft SARPs a single pseudorange error may not be larger than

$$
E_{r, i, \text { max }}=\frac{E_{v, \text { iono, } \max }}{S_{v e r t, i, \max }}=\frac{6.4 \mathrm{~m}}{4}=1.6 \mathrm{~m}
$$

The values assumed in (8) are, however, usually unnecessary conservative. Apart from the $E_{v, \text { iono, } \max }$, as described before, the other conservative assumption is the value of 4 for the $S_{\text {vert }, i \max }$. The ground station is generally unaware of the satellite subset used by the aircraft, and thus the S-factors an airborne receiver may apply. Hence, the most conservative value which can be associated with each satellite has to be used for monitoring purposes. However, depending on the set of satellites, for which GBAS corrections are provided, the $S_{\text {vert }, i \text { max }}$ is limited to values generally smaller than 4 . In order to be conservative a monitoring receiver must determine all possible and valid subsets of satellites for which corrections are provided. Together with conservative assumptions about distance, speed and the aircraft's AAD, a worst case $S_{\text {vert }, i}$ for each satellite can be calculated as maximum over all valid satellite subsets. A subset of satellites is valid if it contains at least four satellites, the $S_{\text {vert }} \leq 4$ limit is fulfilled for each of those satellites and at the same time the Vertical Protection Level (VPL) is smaller than the VAL. From this information the largest projection factor can be calculated for each satellite. This will be done considering the current, given geometry. Only the case of a single affected satellite is considered since a large gradient affecting more than one satellite is considered to be sufficiently unlikely. Due to the fact that the speeds of the ionospheric pierce points of different satellites are practically always different, such a gradient would not appear stationary on different pseudorange measurements (Lee 2011) and could thus be detected by other monitors like the CCD or DSIGMA (Simili 2006, Murphy 2006). By increasing the numerator of (8) and decreasing the denominator at the same time as proposed in this section, the 
actual $E_{r, i, m a x}$ for each satellite will almost always be larger than $1.6 \mathrm{~m}$ and thus relax the currently proposed requirements (ICAO 2010).

194

195

196

197

198

199

200

201

202

203

204

\section{Differential Range Error Monitor}

A method for ensuring this post correction range error limit in general, and mitigating the ionospheric threat in particular, can be based on positioning an additional GNSS receiver at a surveyed location close to the touchdown point of the runway to which approach service is provided. This monitor plays the role of a pseudo user, i.e. it receives and applies GBAS corrections received from the GBAS ground station to correct its own GNSS measurements. The basic idea behind this kind of monitoring is that if an error source, such as an ionospheric disturbance, affects an arriving aircraft in a potentially dangerous way, then the monitor would be affected in a similar way. This is justified since spatial decorrelation between the user and the monitor is minimal, data processing of the raw measurements is the same and the effect does not depend on receiver implementation and airframe characteristics. The smoothed and corrected pseudorange $P_{\text {smt.corr }}$ for each satellite can be described as sum of the theoretical range $r$, an undesired residual range error $E_{\text {range }}$, the user clock bias $c \cdot \Delta t_{\text {user }}$ and a noise term $\eta$ as

$$
P_{\text {smt,corr }}=r+E_{\text {range }}+c \cdot \Delta t_{\text {user }}+\eta
$$

The theoretical range term can be calculated by precise knowledge of the monitor receiver location and is thus a known parameter. The user clock term is common to all pseudorange measurements. It can therefore be removed from the measurements in the same way as it is done in the calculation of the pseudorange corrections in the ground systems. This process is called "smoothed clock adjust" and is described in chapter 3.7.1.2.8.3.5 of ED114A (Eurocae 2013). After removing the geometrical range portion of $P_{\text {smt,corr }}$ for all satellites in view, the average residual range over all satellites is treated as receiver clock bias. While this is usually not exactly true, it does not influence the performance of the monitor since a range bias which is common to all pseudoranges is mapped into the user clock offset. The remaining parts are the sum of the residual range error and noise. These two cannot be separated and their sum shall be called $E_{\text {test }}$, which can be described as

$$
E_{\text {test }}=P_{\text {smt,corr }}-r-\frac{1}{N} \sum_{i=1}^{N} E_{r, i}
$$

with $N$ the number of satellites used. This quantity will be the monitored parameter. 


\section{Monitor design}

In a next step the threshold value for the monitored parameter, i.e. the largest value for which $E_{\text {test }}$ is still considered nominal, has to be derived. This limit has to fulfill the condition that a possible threat is detected with the required probability of missed detection, and at the same time have a sufficiently low probability of false alarm. The trade-off is shown in the following plot.

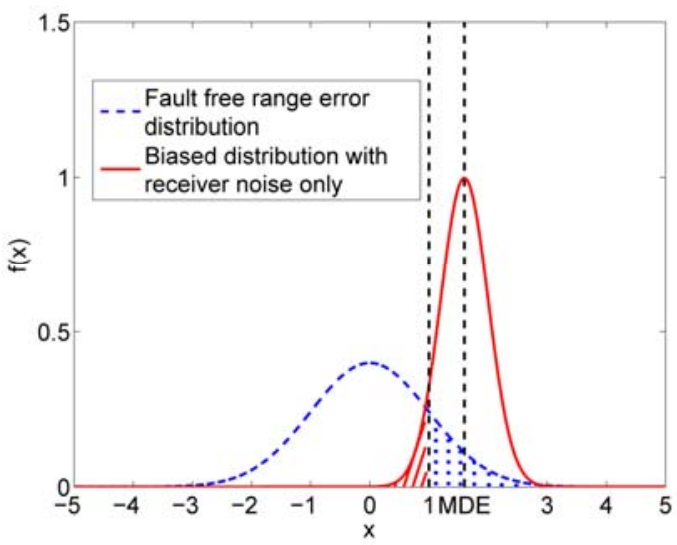

Fig. 4 Example trade-off between probability of missed detection (red shaded) and probability of false alarm (blue dotted). The dashed blue curve shows the expected range error pdf while the red curve shows the expected noise and multipath of the monitoring receiver centered at the minimum detectable error (MDE)

The properties of this monitor depend on four parameters:

- The actual expected distribution of the post-corrected range errors is illustrated by the dashed blue curve in the plot above. In the case of the monitor under discussion it is represented by a non-biased Gaussian distribution for each pseudorange measurement with standard deviation $\sigma_{i}$ and was described in (5). This model depends on geometry, satellite elevation, altitude difference and distance between the airborne receiver and the GBAS reference point, as well as speed of the aircraft and equipment classification. The parameters used in determining the uncertainty should correspond to the expected error distribution of the signal, i.e. for GAST D using $\sigma_{\text {pr_and_30 }}$ from the Type 11 GBAS message (RTCA, 2008a) and a time constant $\tau=30$ seconds to represent the residual noise from the ground system and the appropriate contribution to the ionospheric uncertainty in (5).

- The next influencing parameter is the minimum detectable error (MDE) of the monitor where the red curve of the monitor noise probability density function (pdf) is centered. The MDE in this monitoring 
scheme corresponds to $E_{r, i, \max }$ from (8) for each satellite. As shown earlier, it depends on airworthiness considerations and is the largest tolerable range error which is attributed to one satellite, such that the aircraft can still operate safely.

- Next, the probability of missed detection which is attributed to the monitor has an impact on the overall performance. It is shown as the red shaded area in Figure 4. Since in airworthiness considerations the requirement for the fault case is specified for each error with a probability of occurrence greater than $10^{-9}$, the product of a probability of occurrence and probability of not detecting such a disturbance has to be smaller than $10^{-9}$.

- Finally, the noise and multipath characteristics of the monitoring receiver have a significant impact on the overall performance of the monitor. The lower the noise and multipath characteristics, the larger the monitoring threshold becomes. According to several studies which were performed for evaluation of the GBAS error models, the main concern for ground based receivers is multipath from ground reflections. Hence, strict siting criteria for GBAS reference antennas were developed and multipath limiting antennas are used (FAA 2010).

The expected error distribution is defined in (5). For the derivation of the MDE from (8) we propose to use the actual GPA transmitted in the GBAS message which is typically $3^{\circ}$. When taking a Gaussian noise model for the monitor performance, the detection threshold $E_{\text {monthr }}$ with the respective $p_{m d}$ requirement and the standard deviation of the monitor noise $\sigma$ can be written as

$$
E_{\text {monthr }}=M D E-k_{m d} \cdot \sigma_{\text {monitor }}
$$

with

$$
k_{m d}=-\Phi^{-1}\left(0.5 \cdot p_{m d}\right)=-\sqrt{2} \operatorname{erf}^{-1}\left(p_{m d}-1\right)
$$

and $\Phi(x)$ the standard normal distribution. According to (12) the corresponding missed detection multiplier for $p_{m d}=10^{-9}$ is $k_{m d}=6.1$. The monitoring condition thus becomes

$$
E_{\text {monthr }}<\frac{E_{v, \text { iono,max }}}{S_{\text {vert }, i}}-6.1 \cdot \sigma_{\text {monitor }, i}
$$
probability $p_{\text {iono }}$ can be defined such that instead of requiring a missed detection probability $p_{m d}=10^{-9}$, only the product of prior probability for an ionospheric event and the probability of missed detection has to meet the 
requirement $p_{m d} \cdot p_{\text {iono }}=10^{-9}$. If a meaningful prior probability can be established, e.g. by external monitoring of the state of the ionosphere, we propose to use it in order to achieve better false alarm properties.

For the derivation of the monitoring threshold we propose that the projection factors $S_{\text {vert }, i}$ in (13) should be calculated for each satellite individually as described in the previous section. The resulting largest $S_{\text {vert }, i}$ at each epoch will always be smaller or equal to 4 which leads to relaxed monitoring requirements since $E_{\text {monthr }}$ increases with decreasing $S_{\text {vert }, i}$.

For $\sigma_{\text {monitor }}$ we suggest to use a model which conforms to the GAD C requirement intended for GAST-D operations. We use the same model as used in (RTCA 2008b) which describes the noise as

$$
\sigma_{\text {noise }}=\min \left(0.24 \mathrm{~m}, 0.15 \mathrm{~m}+0.84 \mathrm{~m} \cdot e^{-\theta / 15.8^{\circ}}\right)
$$

It is represented by an elevation dependent function which remains constant for satellite elevation angles below $35^{\circ}$ to reflect the characteristics of multipath limiting antennas (MLAs) and the siting criteria for GBAS. Although the noise restrictions required for this kind of monitoring are the most stringent ones in terms of GBAS Ground Facility Classification, meeting this requirement has been shown to be possible by Dautermann (2012) with choke-ring antennas and standard receivers in a non-optimal environment which does not meet the GBAS siting criteria.

\section{Monitor performance}

In this section an analysis of the different parameters influencing the monitor performance is carried out. Figure 5 shows the minimum gradient which will be detected by this monitor. It is a function of the MDE of the monitor and the distance $d_{\text {monitor }}$ between the GBAS reference point and the location of the monitoring receiver. The minimum slope $g_{\min }$ of a gradient which has to be detected can then be written as

$$
g_{\min }=\frac{M D E}{d_{\text {monitor }}}=\frac{E_{v, \text { iono,max }}}{S_{\text {vert }, i}} \cdot \frac{1}{d_{\text {monitor }}}
$$

with the same notation as in the previous equations. The $S_{\text {vert }, i}$ are calculated according to (7), based on a standard GBAS weighting and assuming an aircraft speed of $70 \mathrm{~m} / \mathrm{s}$, which is a typical approach speed. Out of all satellites in view the $S_{\text {vert }, i}$ for the most limiting satellite, i.e. the satellite with the largest expected test statistic, is taken for a GBAS located at Braunschweig/Wolfsburg airport in northern Germany. The monitor receiver is assumed to be located $5 \mathrm{~km}$ from the GBAS reference point in runway direction towards the east. 
Two different values for the GPA are plotted to show the possible relaxations of the monitoring requirement in comparison to the current $300 \mathrm{~mm} / \mathrm{km}$ limit. The larger GPA relaxes the constraint on the largest tolerable vertical error as described in (1) and thus enlarges the tolerable slope of an ionospheric gradient.

299

300

301

302

303

304

305

306

307

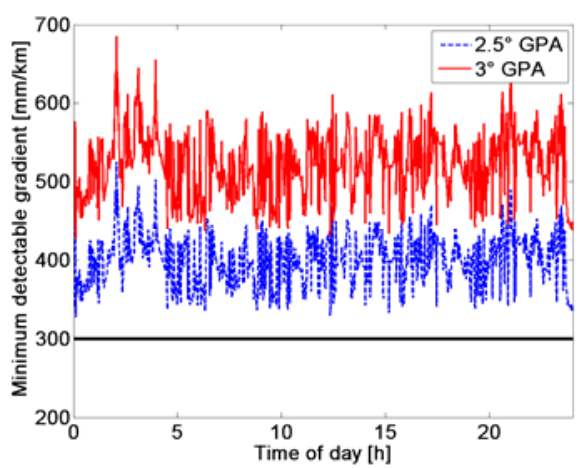

Fig. 5 Minimum size of gradients which will be detected by a monitor located $5 \mathrm{~km}$ away from the GBAS reference point. The dashed blue curve shows the values for a $2.5^{\circ} \mathrm{GPA}$ while the red curve shows the required detection for a standard $3^{\circ}$ GPA. The black line shows the currently required value

The minimum slope of a gradient which needs to be detected considering a $2.5^{\circ} \mathrm{GPA}$ increases to values typically varying between 350 and $450 \mathrm{~mm} / \mathrm{km}$ over a day, while at a $3^{\circ} \mathrm{GPA}$ only gradients as large as 450 to $550 \mathrm{~mm} / \mathrm{km}$ are serious enough to create a potentially dangerous vertical error. At all times the monitoring thresholds are significantly larger than the currently required $300 \mathrm{~mm} / \mathrm{km}$.

Figure 6 shows the corresponding monitoring threshold over one day which was described in (13) with a missed detection probability $p_{m d}=10^{-9}$. For comparison two curves are plotted showing again the different monitoring thresholds for a $2.5^{\circ}$ or $3.0^{\circ} \mathrm{GPA}$. The monitor threshold over the day typically varies between $0.5 \mathrm{~m}$ and $1 \mathrm{~m}$ for the $2.5^{\circ} \mathrm{GPA}$ and between $1 \mathrm{~m}$ and $1.6 \mathrm{~m}$ for the $3^{\circ} \mathrm{GPA}$. The peak shortly before $14 \mathrm{~h}$ represents a situation where for a short time the largest possible $S_{\text {vert }}$ is as small as 2.57 and the corresponding monitor threshold increases to almost $2 \mathrm{~m}$. 


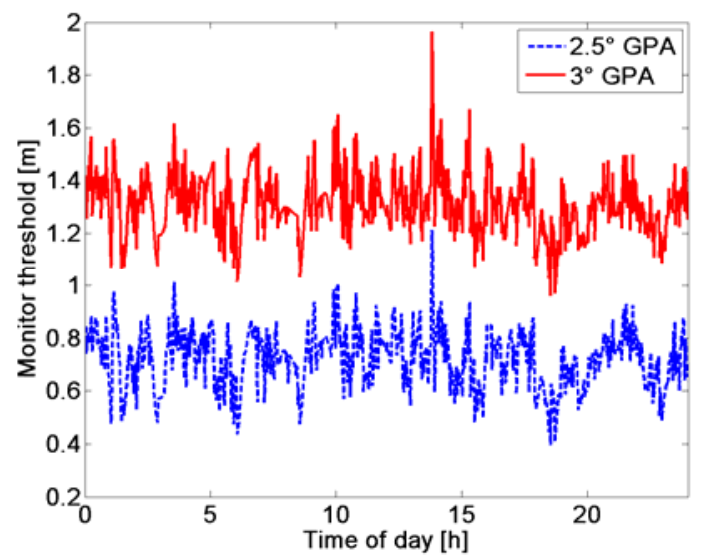

Fig. 6 Monitor thresholds for Braunschweig airport over one day depending on the glide path angle of the approach for the most limiting satellite in view

It can be seen that the higher glide path angle increases the monitor threshold by a factor of roughly 1.6 and thus reduces the false alarm probability of the system accordingly. It is therefore very beneficial to consider the actual GPA for an airport, rather than a general worst approach angle for precision approaches. The false alarm probability is another important parameter since the rate of false alarms impacts the availability and continuity of the system. It is represented as the dotted blue surface in Figure 4 and can be modeled as

$$
p_{f a}=2 \cdot \Phi\left(\frac{-E_{\text {monthr }}}{\sigma_{i}}\right)
$$

with the standard normal distribution $\Phi$, the monitoring threshold $E_{\text {monthr }}$ from (13) and the expected standard deviation of the smoothed, corrected pseudorange $\sigma_{i}$ from (5) for the most critical satellite $i$. Note that Figure 4 only shows the one-sided probability. The factor 2 in (16) takes into account the fact that the errors can be positive or negative. Figure 7 shows the false alarm probability over a day corresponding to the monitoring thresholds from Figure 6 for a $3^{\circ} \mathrm{GPA}$. 


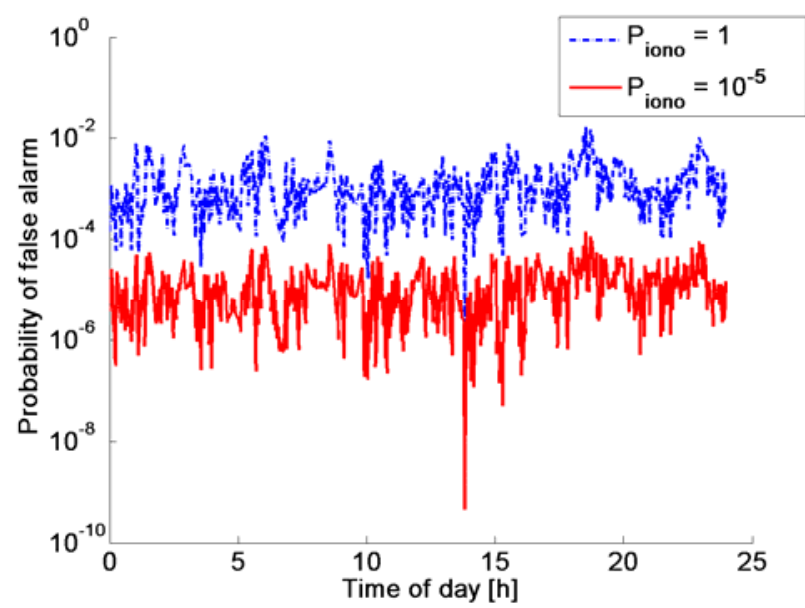

Fig. 7 Probability of false alarm depending on prior probability $\mathbf{P}_{\text {iono }}$ of occurrence of an ionospheric disturbance. Both results assume a $3^{\circ}$ GPA for the approach

The dashed blue curve shows the case if no prior probability for the occurrence of an ionospheric disturbance is defined. In this case the false alarm probability mostly stays in the range of $10^{-2}$ to $10^{-5}$. For an operational system these values are large, despite the fact that flagging one satellite would not necessarily limit the availability of the whole GBAS, since usually there are between 6 and 12 satellites available for navigation. However, if some credit is taken for the fact that these storms happen very rarely, performance of this monitor improves significantly. Previous work on this issue, such as (Khanafseh 2012) or (Belabbas 2012) used a value of $10^{-5}$ for the assumed probability of occurrence. This limits the required probability of missed detection to just $10^{-4}$ and reduces the k-factor in (11) from 6.1 to 3.7 and thus relaxes the monitoring problem. The results for the false alarm probability assuming this prior probability for an ionospheric disturbance is shown in red in Figure 7. It decreases down to values in the range of $10^{-4}$ to $10^{-6}$. However, a standardized way of determining such a probability has not yet been developed and needs to be investigated. Due to the more frequent observation of ionospheric storms, scintillations and plasma bubbles in the high and low latitudes as opposed to the midlatitudes, such a probability should be defined locally together with the ionospheric threat model or determined by external information, such as ionospheric parameters from a space based augmentation system (SBAS).

The results shown above are an example for performance at a certain location. However, the monitoring thresholds and the associated false alarm probabilities do not change substantially in different locations around the earth and at different latitudes. This is a result of the selection of the worst possible geometry at any location for the derivation of a conservative monitor threshold. Figure 8 shows the simulated thresholds over one day for three selected airports in different regions. 


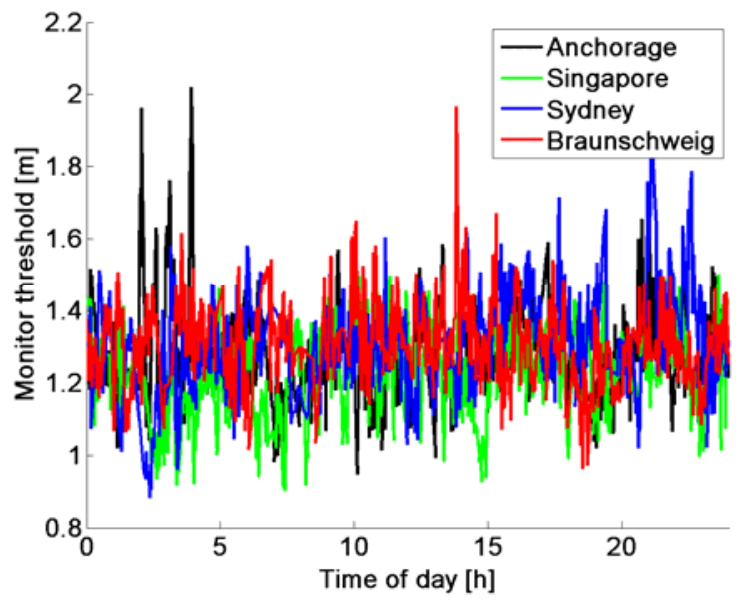

Fig. 8 Monitor thresholds for the most limiting satellite in view over one day at different latitudes. Anchorage as example for a polar region airport (Latitude $\left.61^{\circ} \mathrm{N}\right)$, Singapore for an equatorial airport $\left(1^{\circ} \mathrm{N}\right)$ and Sydney as example for mid-latitude $\left(33^{\circ} \mathrm{S}\right)$ compared to Braunschweig assuming a $3^{\circ} \mathrm{GPA}$

Anchorage is located at a northern latitude of $61^{\circ}$, Singapore is located very close to the equator at $1^{\circ}$ North and Sydney is located at $33^{\circ} \mathrm{S}$ in the equatorial to mid-latitude region. Together with Braunschweig (at $52^{\circ} \mathrm{N}$ ) those airports represent the typical range in which GBAS stations are expected to be located. The monitor thresholds tend to be the lowest in Singapore (green curve). The times when the monitoring threshold decreases below $1 \mathrm{~m}$ for the most critical satellite correspond to epochs when many satellites are visible (13 or 14). At these epochs it is possible to select subsets which create larger values for $S_{\text {vert }}$ than at times when there are fewer satellites available. Sydney shows a monitoring threshold very similar to that of Braunschweig. Only for one short period of about 20 minutes around $2 \mathrm{~h} 30 \mathrm{~min}$ it drops below a value of $1 \mathrm{~m}$. During this time there are also 13 satellites visible while at most other epochs the number is mostly between 12 and 8 . For Anchorage there are four spikes visible between $2 \mathrm{~h}$ and $4 \mathrm{~h}$ where the threshold becomes very large. At these epochs there are 11, 9, 7 and 10 satellites visible while most of the time there are at least 10 satellites available, sometimes up to 14 . In a similar way as could be observed at the other locations, a smaller number of available satellites generally increases the monitoring threshold. However, the effect is not as visible as in the other example sites because due the location far up north more satellite subsets are excluded in the geometry screening process.

We showed a method to meet the monitoring requirements for an automatic landing system based on GBAS navigation from an airworthiness perspective. Instead of specific requirements concerning ionospheric gradients only, it targets the actual requirement of ensuring a safe landing in the touchdown box given the current actual 
376 satellite constellation and approach geometry. The detection threshold can be increased compared to the draft

377 SARPS by modifying some overly conservative assumptions and taking into account the actual satellite 378 geometry. This method has the advantage of significantly relaxing the ground monitoring requirement for

379 ionospheric disturbances. Furthermore, any errors which do not relate to receiver implementation and airframe 380 characteristics of arriving aircraft can be detected by placing the monitoring receiver close to the touchdown

381 point, irrespective of their source. This method makes the siting limit of $5 \mathrm{~km}$ irrelevant because it can limit 382 potential differential ranging errors to a safe level, irrespective of the distance to the GBAS ground station.

383 Reference receiver siting at airports is a very challenging task because of required obstacle clearance near 384 runways and taxiways, as well as protection of the reference antennas against potential sources of multipath. The 385 elimination or relaxation of the $5 \mathrm{~km}$ limit would simplify reference receiver siting significantly and make 386 GBAS usable at more airports. At large capacity and environmentally constrained airports GBAS is expected to 387 provide approach service on advanced and possibly curved trajectories and thus to contribute to reduce emissions 388 and increase capacity. The monitoring receiver can furthermore check the validity and provide independent 389 monitoring of the broadcast corrections and runway reference coordinates by means of its known position 390 relative to the approach track.

\section{Acknowledgment}

393 Michael Felux' activities were conducted within the internal projects TOPGAL and GRETA. Jiyun Lee was 394 supported by MLTM under grant 10AVI-NAV01 


\section{References}

Belabbas B and Meurer M (2012) Carrier Phase and Code Based Absolute Slant Ionosphere Gradient Monitor for GBAS, Proc. ION GNSS 2012, Institute of Navigation, Nashville, TN, USA, pp. 2201-2208

Boeing (2005), Determining the Vertical Alert Limit Requirements for a Level of GBAS Service that is Appropriate to Support CAT II/III Operations, D6-83447-4

Burns J, Clark B, Cassell R, Shively C, Murphy T, Harris M, (2009), Conceptual Framework for the Proposal for GBAS to Support CAT III Operations, ICAO NSP WGW November 2009

Clark B, DeCleene B, (2006) Alert Limits: Do we Need Them for CAT III? Deriving GBAS Requirements for Consistency with CAT III Operations, Proc. ION GNSS 2006, Institute of Navigation, Fort Worth, TX, USA, pp. 3070-3081

Dautermann T, Felux M, Grosch A (2012) Approach service type D evaluation of the DLR GBAS testbed. GPS SOL. doi: 10.1007/s10291-011-0239-3.

Eurocae, (2013), Minimum Operational Performance Specification for Global Navigation Satellite Ground Based Augmentation System Ground Equipment to Support Category I Operations, ED-114A, EUROCAE.

European Aviation Safety Agency (EASA), (2003), Certification Specifications for All Weather Operations (CSAWO)

Federal Aviation Administration (FAA), (1999), Criteria for Approval of Category III Weather Minima for Take-off, Landing, and Rollout (AC 120-28D), US Department of Transportation, Washington, DC, USA

Federal Aviation Administration (FAA), (2010), Siting Criteria for Ground Based Augmentation Systems (GBAS), Order 6884.1, US Department of Transportation, Washington, DC, USA

Fujita S, Takayuki Y, Saito S, (2010) Determination of Ionosphere Gradient in Short Baselines by Using Single Frequency Measurements. J AERO ASTRO AVI, A-42:269-275

Harris M, Murphy T, (2007), Geometry Screening for GBAS to Meet CAT III Integrity and Continuity Requirements, Proc. ION NTM 2007, Institute of Navigation, San Diego, CA, USA, pp. 1221-1233

ICAO (2010), GBAS CAT II/III Development Baseline SARPs - Draft proposed changes to Annex 10, Volume I, ICAO NSP WGW 
Jung S, Lee J, (2012), Long-term ionospheric anomaly monitoring for ground based augmentation systems, RADIO SCI, 47, RS4006, doi:10.1029/2012RS005016.

Khanafseh S, Pullen S, Warburton J, (2012) Carrier phase ionospheric gradient ground monitor for GBAS with experimental validation. Navigation 59: 51-60. doi: 10.1002/navi.3.

Lee J, Seo J, Par YS, Pullen S, Enge P (2011) Ionospheric threat mitigation by geometry screening in GroundBased Augmentation Systems. J Aircraft 48(4):1422-1433. doi:10.2514/1.C031309

Mayer C, Belabbas B, Jakowski N, Meurer M, Dunkel W, (2009), Ionosphere threat space model assessment for GBAS, Proc. ION GNSS 2009, Institute of Navigation, Savannah, GA, USA, pp. 1091-1099

McGraw GA, Murphy T, Brenner M, Pullen S, Van Dierendonck AJ, (2000) Development of the LAAS accuracy models. Proc. ION GPS 2000, Institute of Navigation, Salt Lake City, UT, USA, pp. 1212-1223

Murphy T, Harris M, (2006), Mitigation of the Ionospheric Gradient Threats for GBAS to Support CATII/III, Proc. ION GNSS 2006, Institute of Navigation, Fort Worth, TX, USA, pp. 449-461

Pullen S, Park YS, Enge P, (2009) Impact and mitigation of ionospheric anomalies on ground-based augmentation of GNSS, RADIO SCI, 44, RS0A21, doi:10.1029/2008RS004084.

Schuster W, Washington O, (2010) Harmonisation of Category-III Precision Approach Navigation System Performance Requirements. J Navigation, doi:10.1017/S0373463310000287

Simili DV, Pervan B. (2006), Code-carrier divergence monitoring for the GPS local area augmentation system, Proc. ION/IEEE Position, Location, and Navigation Symposium 2006, pp. 483-493, ION/IEEE, San Diego, CA, USA

RTCA (2008a), GNSS-Based Precision Approach Local Area Augmentation System (LAAS) Signal-in-Space Interface Control Document (ICD), RTCA DO-246D, Washington, DC.

RTCA (2008b), Minimum Operational Performance Standards for GPS Local Area Augmentation System Airborne Equipment, RTCA DO-253C, Washington, DC. 
472 Michael Felux is a research associate at the German Aerospace Center (DLR). He graduated in technical 473 mathematics at Technische Universität München in 2009. The same year he joined DLR specializing on GBAS 474 integrity issues for CAT II/III operations. He is active in different GBAS working groups.

475 Jiyun Lee is an Associate Professor in Aerospace Engineering at Korea Advanced Institute of Science and 476 Technology. She has supported the GBAS and SBAS program over the past seven years as a consulting 477 professor at Stanford University and a Principal GPS Systems Engineer at Tetra Tech AMT. She received her 478 Ph.D. from Stanford University in aeronautics and astronautics.

479 Florian Holzapfel is a senior Member of AIAA, Member DGLR and a Full Professor and Director of the 480 Institute of Flight System Dynamics of Technische Universität München (TUM). He received his Doctorate from 481 TUM working on nonlinear adaptive controls of unmanned aerial vehicles. He is chairman of the DGLR Flight 482 Control TC and a member of the GNC TCs of CEAS and AIAA. Furthermore, he serves on the senate of DGLR. 